Check for updates

Cite this: Phys. Chem. Chem. Phys. 2022, 24, 7387

Received 12th January 2022 , Accepted 18th February 2022

DOI: $10.1039 / \mathrm{d} 2 \mathrm{cp} 00180 \mathrm{~b}$

rsc.li/pccp

\section{An $a b$ initio study of the photodissociation of the vinyl radical}

\author{
A. Bouallagui, ${ }^{a b}$ A. Zanchet, (iD ${ }^{b}$ L. Bañares ${ }^{c d}$ and A. García-Vela (D) *b
}

Photodissociation of the vinyl radical through pathways $\mathrm{CH}_{2} \mathrm{CH} \rightarrow \mathrm{CH}_{2} \mathrm{C}+\mathrm{H}, \mathrm{CH}_{2} \mathrm{CH} \rightarrow \mathrm{CHCH}+\mathrm{H}$, and $\mathrm{CH}_{2} \mathrm{CH} \rightarrow \mathrm{CH}_{2}+\mathrm{CH}$ is investigated by means of high-level ab initio calculations. Potential-energy curves (PECs) along the corresponding dissociating bond distance associated with the ground and several excited electronic states involved in the above fragmentation pathways, as well as the nonadiabatic couplings connecting the different states, are obtained. The findings of several experiments on vinyl photodissociation performed at different excitation wavelengths are analyzed and explained qualitatively in the light of the present PECs. A two-dimensional representation (consisting of radial and angular coordinates to represent one of the $\mathrm{H}$ atoms of the $\mathrm{CH}_{2}$ group) is also used to calculate the electronic states. The surfaces obtained reflect a rich variety of conical intersections, exit barriers, and nonadiabatic couplings leading to predissociation in different regions of energy and of the two coordinates, suggesting a complex photodissociation dynamics of the $\mathrm{CH}_{2} \mathrm{CH} \rightarrow \mathrm{CHCH}+\mathrm{H}$ pathway, with rather different fragmentation mechanisms involved. The two-dimensional results also provide interesting information on the mechanism of in-plane hydrogen migration from the $\mathrm{CH}_{2}$ group to the $\mathrm{CH}$ one through a high-lying transition state.

\section{Introduction}

The vinyl radical (VR), $\mathrm{H}_{2} \mathrm{C}_{\beta}=\mathrm{C}_{\alpha} \mathrm{H}$, is known to be a highly reactive species which is the simplest open-shell olefinic radical. The VR plays an important role as an intermediate in several areas of chemistry, including hydrocarbon combustion $^{1-3}$ and hydrocarbon plasma chemistry, ${ }^{4}$ the chemistry of planetary atmospheres, ${ }^{5-9}$ and as a precursor for the formation of soot. ${ }^{10-12}$ This radical presents two equivalent global minima corresponding to the planar structure of $C_{\mathrm{s}}$ symmetry, where the $\mathrm{C}_{\alpha} \mathrm{H}$ group is bent with respect to the $\mathrm{C}-\mathrm{C}$ axis. The two global minima are separated by barriers with three different saddle points on top. The lowest energy saddle point has a Y-shaped planar structure of $C_{2 v}$ symmetry. Two additional saddle points lie at (a similar) much higher energy, one being a planar bridged structure of $C_{2 \mathrm{v}}$ symmetry, and the other one, with one $\mathrm{H}$ atom of $\mathrm{C}_{\beta} \mathrm{H}_{2}$ forming an out-of-plane bridged structure between the two carbon atoms and the

\footnotetext{
${ }^{a}$ Laboratoire de Spectroscopie Atomique, Moléculaire et Applications-LSAMA LR01ES09, Faculté des Sciences de Tunis, Université de Tunis El Manar, 2092, Tunis, Tunisia

${ }^{b}$ Instituto de Física Fundamental, Consejo Superior de Investigaciones Cientificas, Serrano 123, 28006 Madrid, Spain. E-mail: garciavela@iff.csic.es

${ }^{c}$ Departamento de Química Física, Facultad de Ciencias Químicas, Universidad Complutense de Madrid (Unidad Asociada I + D + i CSIC), 28040 Madrid, Spain

${ }^{d}$ Instituto Madrileño de Estudios Avanzados en Nanociencia (IMDEA-Nanoscience), 28049 Madrid, Spain
}

remaining two $\mathrm{H}$ atoms. The two potential wells are connected by a tunneling path, through which one $\mathrm{H}$ atom, either in the $\mathrm{C}_{\alpha} \mathrm{H}$ or in the $\mathrm{C}_{\beta} \mathrm{H}_{2}$ group, undergoes migration to the other equivalent well through one of the above transition states. Most of the research efforts have concentrated so far on investigating the tunneling path occurring through the lowest-lying $C_{2 \mathrm{v}}$ inplane saddle point, where the $\mathrm{H}$ atom of $\mathrm{C}_{\alpha} \mathrm{H}$ undergoes a largeamplitude migration from one bent position to the other equivalent one, but without swapping the carbon atom to which it remains bonded. Hydrogen migration through the other two high-lying saddle points involves swapping of the carbon atom and thus of the radical center.

Several experimental studies have reported measurements of absorption bands to different electronic states of the VR in the last few decades. ${ }^{11,13-20}$ However, much of the experimental work has dealt with rovibrational spectroscopy of the double well and the tunneling dynamics of the VR in the ground $\tilde{X}^{2} A^{\prime}$ electronic state through the lowest-lying transition state. In this sense, infrared spectra ${ }^{21-23}$ and tunneling splittings of the VR have been reported..$^{21,24,25}$ In addition to spectroscopic experiments, the photodissociation dynamics of the VR was investigated experimentally at $243 \mathrm{~nm}$ by means of velocity map imaging (VMI). ${ }^{26}$ At this excitation wavelength the two first excited electronic states, $\tilde{A}^{2} A^{\prime \prime}$ and $\tilde{B}^{2} A^{\prime \prime}$, can be accessed, consistent with the anisotropy found in the velocity map images that resembles perpendicular transitions. More recently, the photodissociation of the VR at $199 \mathrm{~nm}$ was also 
explored through the VMI technique. ${ }^{27}$ Photodissociation of vinyl was also observed in time-of-flight spectra at $193 \mathrm{~nm}$ as a secondary process, after the primary photodissociation of ethylene. ${ }^{28}$ In this case, the VR fragment produced after the first dissociation of ethylene would absorb a second photon, dissociating into $\mathrm{H}_{2} \mathrm{CC} / \mathrm{HCCH}$ products.

From the theoretical point of view, several ab initio calculations on the VR have been published in the literature, which in general deal with the ground electronic state. ${ }^{29-39}$ Only a few works studied the low-lying excited electronic states, ${ }^{34,35}$ and they reported optimized geometries and vertical excitation energies, but not potential-energy surfaces or curves. The ground state calculations are typically reduced-dimensionality representations; some of them are even one-dimensional (1D) potential curves that are used to calculate 1D tunneling splittings. ${ }^{38,39}$ At present only two full-dimensional ab initio calculations have been reported for the ground $\tilde{X}^{2} A^{\prime}$ electronic state of the VR. ${ }^{40,41}$ By using these two surfaces, fulldimensional vibrational energy levels and tunneling splittings have been computed. ${ }^{42-44}$ While the agreement between the results of the three calculations performed is quite good in general, the results of Yu et al. ${ }^{42}$ and Wang et al. ${ }^{44}$ exhibit an overall better agreement.

It is clearly apparent from the literature that both the experimental and the theoretical research studies on the VR have concentrated on the ground electronic state. More specifically, the focus has been mostly on studying the tunneling dynamics through the lowest-lying in-plane transition state. This implies that the available potential-energy surfaces are essentially restricted to the $\tilde{X}^{2} A^{\prime}$ electronic state, and in the interaction region where the tunneling mechanism takes place. Thus, little is known about the excited electronic states of vinyl, both in the interaction and in the asymptotic regions, which makes it very difficult to investigate the photodissociation dynamics of this radical. In addition, knowledge of the tunneling dynamics through the other two high-lying transition states is also very scarce.

The present work intends to shed light on the structure of the excited electronic states of the VR in order to understand its photodissociation dynamics. There are three possible photodissociation pathways:

$$
\begin{aligned}
& \mathrm{CH}_{2} \mathrm{CH} \rightarrow \mathrm{CH}_{2} \mathrm{C}+\mathrm{H} \\
& \mathrm{CH}_{2} \mathrm{CH} \rightarrow \mathrm{CHCH}+\mathrm{H} \\
& \mathrm{CH}_{2} \mathrm{CH} \rightarrow \mathrm{CH}_{2}+\mathrm{CH}
\end{aligned}
$$

A possible additional fragmentation pathway is $\mathrm{CH}_{2} \mathrm{CH} \rightarrow$ $\mathrm{CCH}+\mathrm{H}_{2}$, although it would involve a roaming mechanism of one $\mathrm{H}$ atom and the breaking of two $\mathrm{C}-\mathrm{H}$ bonds, and thus it is expected to have associated a low probability. The equivalent pathway leading to $\mathrm{C}_{2} \mathrm{H}_{3}+\mathrm{H}_{2}$ was investigated theoretically in ethyl, ${ }^{45}$ indicating that its effect was actually minor. In this work, the dissociation pathways (1)-(3) have been investigated by means of multireference configuration interaction (MRCI) $a b$ initio calculations. The potential-energy curves (PEC) of the ground and several excited electronic states along the corresponding dissociation coordinates of the three fragmentation pathways have been calculated. The conical intersections (CI) between different excited electronic states have been identified, and in some cases the couplings associated with these CIs have been computed. The nonadiabatic couplings between states leading to predissociation have also been obtained. In addition to the 1D representation employed for the fragmentation pathways (1)-(3), we have used a two-dimensional (2D) representation to investigate pathway (2). In this case, the dissociation coordinate $R$ is the distance between one $\mathrm{H}$ atom of the $\mathrm{C}_{\beta} \mathrm{H}_{2}$ group and the $\mathrm{HC}-\mathrm{CH}$ center of mass, and the additional coordinate is the angle $\theta$ formed by the dissociation coordinate and the $\mathrm{H}-\mathrm{C}-\mathrm{C}-\mathrm{H}$ axis. This $2 \mathrm{D}$ representation of the potentialenergy surfaces allows us to explore the angular dependence of the ground and excited electronic states of VR, which for $\theta=90^{\circ}$ corresponds to the high-lying in-plane bridged $C_{2 \mathrm{v}}$ transition state.

\section{Methodology}

The potential energy curves of the ground and several excited electronic states of vinyl have been computed at the multireference configuration interaction (MRCI) level $^{46}$ (without Davidson correction) for different reaction coordinates. A relaxed scan (i.e., a geometry optimization on all the coordinates except the one of interest) on the ground state was performed along both the $\mathrm{C}-\mathrm{H}$ and $\mathrm{C}-\mathrm{C}$ stretching coordinates associated with dissociation through pathways (1)-(3). In the case of the $2 \mathrm{D}$ representation used, the relaxed scan was performed as a function of the Jacobi angle (i.e., the angle between the $\mathrm{C}-\mathrm{C}$ axis and the vector joining one $\mathrm{H}$ atom of the $\mathrm{CH}_{2}$ group and the center of mass of the $\mathrm{C}-\mathrm{C}$ bond). The relaxed scans were performed at the CASPT2 level ${ }^{47}$ to properly describe the breaking of the different bonds, as mono-reference methods usually fail to describe these types of processes.

The MRCI calculations were performed over the optimized geometries obtained to build the potential-energy curves of the first electronic states and to compute the nonadiabatic coupling matrix elements (NACMEs). The NACMEs were calculated following the DDR procedure available in the Molpro package. The procedure consists of performing a three-point numerical derivative of the transition density matrices considering $\langle\gamma(R) \mid \gamma(R)\rangle,\langle\gamma(R) \mid \gamma(R+D R)\rangle$, and $\langle\gamma(R) \mid \gamma(R-D R)\rangle$, where $R$ is the reaction coordinate considered. This is carried out for each pair of electronic states considered. For the sake of accuracy, the value $\mathrm{DR}=10^{-4} \AA$ was used.

The orbitals and references used in the MRCI calculations were obtained by previous state-average $\mathrm{CASSCF}^{48}$ calculations including six states of $A^{\prime}$ symmetry and six states of $A^{\prime \prime}$ symmetry of the $C_{\mathrm{s}}$ group (genuine to vinyl). The chosen active space includes five $a^{\prime}$ orbitals and three $a^{\prime \prime}$ orbitals, while the two orbitals corresponding to the 1s orbitals of the carbon atoms were not included. With the active space chosen the number of configuration Slater functions (CSFs) is 13916 and 
13804 for the $A^{\prime}$ and $A^{\prime \prime}$ symmetries, respectively. This active space is stable along all the reaction coordinates for both $\mathrm{H}$ atom elimination channels, keeping the orbitals and allowing a smooth behavior of the electronic wavefunction. Due to the different nature of the bonding, this active space is not optimal to describe the $\mathrm{C}-\mathrm{C}$ breaking and a switch in orbitals in the long range region gives rise to some irregularities in the description of the electronic wavefunction. Despite this problem, we still used this active space to study this channel in order to keep the homogeneity of the calculation and to be able to compare the results of this channel with those of the $\mathrm{H}$-atom elimination ones. This choice is further justified by the fact that these convergence instabilities occur far from the FranckCondon region, and do not affect the physical interpretation. Nevertheless, less electronic states were considered for this channel at the MRCI level, where only four states of $A^{\prime}$ symmetry and four states of $A^{\prime \prime}$ symmetry were included. The augcc-pVTZ basis set was employed for all the calculations using the MOLPRO package. ${ }^{49}$

\section{Results and discussion}

\subsection{The different dissociation pathways of the vinyl radical}

Alkyl radical molecules like vinyl typically present different fragmentation pathways upon photoexcitation depending on the specific bond of the molecule which is broken. The ethyl radical, $\mathrm{C}_{\beta} \mathrm{H}_{3}-\mathrm{C}_{\alpha} \mathrm{H}_{2}$, can be considered the saturated counterpart of VR, and recently dissociation pathways similar to (1)-(3), namely $\mathrm{H}$-atom elimination from the $\mathrm{C}_{\beta} \mathrm{H}_{3}$ and $\mathrm{C}_{\alpha} \mathrm{H}_{2}$ groups $^{27,50,51}$ and $\mathrm{C}-\mathrm{C}$ bond cleavage, ${ }^{52}$ have been investigated both experimentally and theoretically. The calculated $a b$ initio PECs of the ground and excited electronic states, and the couplings between them for the different dissociation pathways helped rationalize the experimental results and the photodissociation mechanisms. ${ }^{27,50-52}$ The PECs of the different electronic states reported here for pathways (1)-(3) of VR also intend to provide a guide to interpret currently available and future photodissociation experiments.

Fig. 1-3 display the $a b$ initio calculated adiabatic PECs corresponding to the ground and first excited electronic states, along with the associated nonadiabatic couplings between them for the dissociation pathways (1)-(3). Let us first discuss the two $\mathrm{H}$-atom elimination pathways (1) and (2), which have been studied experimentally more extensively. Fig. 1a displays the PECs corresponding to pathway (1), which includes $\mathrm{H}$-fragment elimination from the $\mathrm{C}_{\alpha} \mathrm{H}$ group producing vinylidene, $\mathrm{CH}_{2} \mathrm{CH} \rightarrow \mathrm{CH}_{2} \mathrm{C}+\mathrm{H}$. Several excited state curves exhibit exit barriers to dissociation as a consequence of avoided crossings with other electronic states, caused by the nonadiabatic couplings shown in Fig. 1b. Such barriers play an important role in different photodissociation mechanisms involved in pathway (1). Several possible conical intersections have been identified. There are two CIs at short distances $\left(R_{\mathrm{C}-\mathrm{H}}<1.5 \AA\right.$, indicated by blue circles), one between the $\tilde{E}^{2} A^{\prime}$ and $\tilde{G}^{2} A^{\prime}$ electronic states and the other one between the $\tilde{D}^{2} A^{\prime}$ and $\tilde{E}^{2} A^{\prime}$
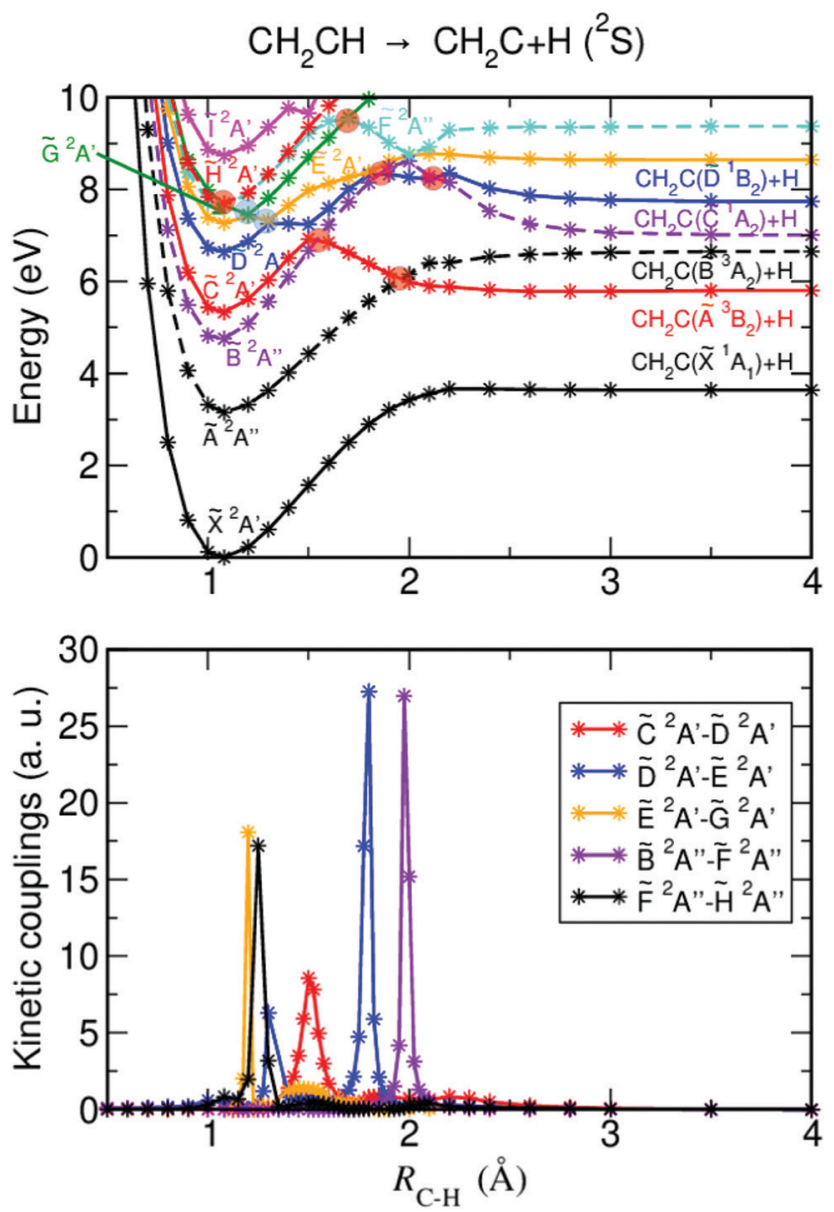

Fig. 1 (a) Adiabatic potential-energy curves of different electronic states along the $\mathrm{C}-\mathrm{H}$ bond distance for the $\mathrm{CH}_{2} \mathrm{CH} \rightarrow \mathrm{CH}_{2} \mathrm{C}+\mathrm{H}$ fragmentation pathway. The blue circles denote the $\mathrm{Cls}$ identified within the planar $\mathrm{C}_{\mathrm{s}}$ symmetry while the orange circles denote $\mathrm{Cls}$ that are expected to occur on leaving the plane of the $C_{s}$ symmetry. (b) Calculated nonadiabatic couplings between the different electronic states that include the couplings (in low resolution) associated with the two $\mathrm{Cls}$ labeled by the blue circles, at $R_{\mathrm{C}-\mathrm{H}}<1.5 \AA$ (between the $\tilde{E}^{2} A^{\prime}$ and $\tilde{G}{ }^{2} A^{\prime}$ states and the $\tilde{D}^{2} A^{\prime}$ and $\tilde{E}^{2} A^{\prime}$ states), and the couplings leading to predissociation.

electronic states. The couplings associated with these two CIs are shown in Fig. 1b in low resolution (meaning that the curves of the CI couplings do not contain enough points to reach the maximum coupling intensity). Another six CIs (indicated by orange circles) are identified in the form of crossings between states of ${ }^{2} A^{\prime}$ and ${ }^{2} A^{\prime \prime}$ symmetry within the planar $C_{\mathrm{s}}$ symmetry assumed in the present calculations. These CIs are expected to occur upon leaving the plane of the $C_{\mathrm{s}}$ symmetry. Thus, several CIs between excited electronic states are found that can determine to a large extent the photodissociation mechanisms, although most of them are located at rather high energies, implying that they will operate only for relatively high excitation energies.

It is noted that some discontinuities may appear in the highest-lying electronic states displayed in Fig. 1-3 (and particularly in the $\tilde{I}^{2} A^{\prime}$ state of Fig. 1). Such discontinuities can be attributed to a kind of "border effect" caused by avoided 

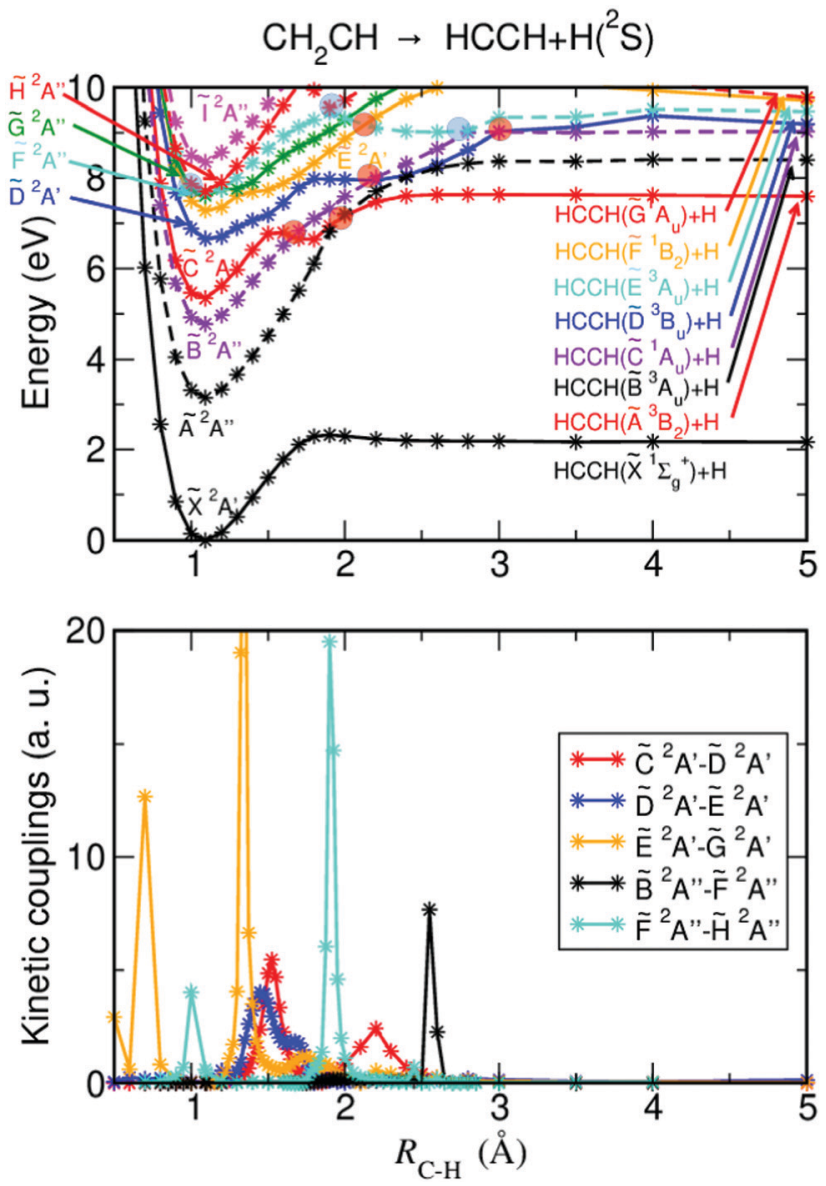

Fig. 2 (a) Adiabatic potential-energy curves of the different electronic states along the $\mathrm{C}-\mathrm{H}$ bond distance for the $\mathrm{CH}_{2} \mathrm{CH} \rightarrow \mathrm{CHCH}+\mathrm{H}$ fragmentation pathway. The blue circles denote the $\mathrm{Cls}$ identified within the planar $C_{s}$ symmetry while the orange circles denote $\mathrm{Cls}_{\mathrm{s}}$ that are expected to occur upon leaving the plane of the $C_{s}$ symmetry. (b) Calculated nonadiabatic couplings between the different electronic states that include the couplings (in low resolution) associated with the two Cls labeled by the blue circles at $R_{\mathrm{C}-\mathrm{H}}<2.0 \AA$ (between the $\tilde{E}^{2} A^{\prime}$ and $\tilde{G}^{2} A^{\prime}$ states and the $\tilde{F}^{2} A^{\prime \prime}$ and $\tilde{H}^{2} A^{\prime \prime}$ states), and the couplings leading to predissociation.

crossings between higher-lying electronic states that were not included in the calculation and the last, highest-lying state reported. Still the discontinuities found are rather small.

The PECs associated with $\mathrm{H}$-atom elimination from the $\mathrm{C}_{\beta} \mathrm{H}_{2}$ group, producing acetylene $(\mathrm{HC}=\mathrm{CH})$ through pathway (2), are displayed in Fig. 2a, and the corresponding nonadiabatic couplings between different electronic states are shown in Fig. 2b. There are two main differences between the curves of Fig. 2a and those of Fig. 1a. The first difference is that while some excited states in Fig. 2a also display exit barriers to dissociation, such barriers are remarkably lower in general than those of the curves of Fig. 1a. The second difference is that the ground state asymptote is much more separated in energy from the asymptotes of the low-lying excited states than that shown in Fig. 1a. Indeed, the separation of the ground and first excited state for $\mathrm{CH}_{2} \mathrm{C}+\mathrm{H}$ dissociation is $\sim 2 \mathrm{eV}$, while for fragmentation into $\mathrm{CHCH}+\mathrm{H}$ is about $6 \mathrm{eV}$. The implication is
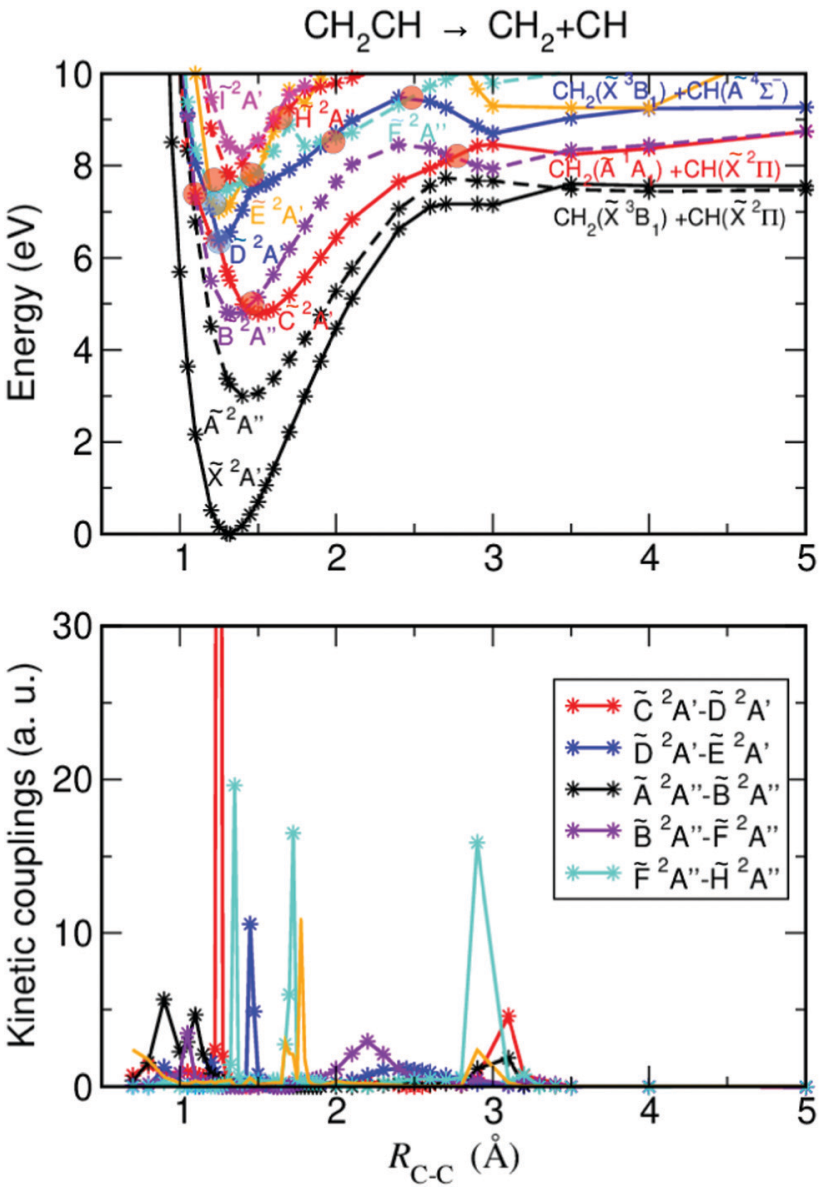

Fig. 3 (a) Adiabatic potential-energy curves of the different electronic states along the $\mathrm{C}-\mathrm{C}$ bond distance for the $\mathrm{CH}_{2} \mathrm{CH} \rightarrow \mathrm{CH}_{2}+\mathrm{CH}$ fragmentation pathway. The blue circles denote the $\mathrm{Cls}$ identified within the planar $C_{s}$ symmetry while the orange circles denote $\mathrm{Cls}$ that are expected to occur upon leaving the plane of the $C_{\mathrm{s}}$ symmetry. The state $\tilde{G}{ }^{2} A^{\prime}$ is missing because it was not included in the calculation in order to avoid convergence problems. The vinyl states $\tilde{X}^{2} A^{\prime}$ and $\tilde{A}{ }^{2} A^{\prime \prime}$ correlate asymptotically with the same $\mathrm{CH}_{2}\left(\tilde{X}^{3} B_{1}\right)+\mathrm{CH}\left(\tilde{X}^{2} \Pi\right)$ fragments, while the vinyl states $\tilde{B}^{2} A^{\prime \prime}$ and $\tilde{C}^{2} A^{\prime}$ correlate asymptotically with the same $\mathrm{CH}_{2}(\tilde{A}$ $\left.{ }^{1} A_{1}\right)+\mathrm{CH}\left(\tilde{X}^{2} \Pi\right)$ fragments. (b) Calculated nonadiabatic couplings between the different electronic states that include the couplings (in low resolution) associated with the two $\mathrm{Cls}$ labeled by the blue circles at $R_{\mathrm{C}-\mathrm{C}}<1.5 \AA$ (between the $\tilde{C}^{2} A^{\prime}$ and $\tilde{D}^{2} A^{\prime}$ states and the $\tilde{D}^{2} A^{\prime}$ and $\tilde{E}^{2} A^{\prime}$ states), and the couplings leading to predissociation. The intensity of one $\mathrm{Cl}$ coupling has been cut in order to facilitate visualization of the remaining couplings.

that for relatively low excitation energies (below the lowest excited state asymptote) only acetylene in the ground electronic state, $\operatorname{CHCH}\left(\tilde{X}^{1} \Sigma_{g}^{+}\right)$, will be produced, and the dominant photodissociation mechanism will involve the internal conversion from the initially populated excited state to the ground one.

In the following we will discuss briefly the three main photodissociation experiments of $\mathrm{VR},{ }^{26-28}$ which are carried out at different excitation energies, in the light of the PECs of Fig. 1 and 2. In the experiments of Suits and co-workers, ${ }^{26}$ excitation at $\lambda=243 \mathrm{~nm}$ of cold radicals (i.e. low internal energy), produced by the photolysis of vinyl chloride in helium 
at $193 \mathrm{~nm}$ in the expansion prior to collimation through a skimmer, allows us to access to the two first excited states, $\tilde{A}^{2} A^{\prime \prime}$ and $\tilde{B}^{2} A^{\prime \prime}$. The main contribution of the measured $\mathrm{H}$-fragment translational energy distribution (TED) exhibited a Boltzmanntype shape with a peak at $\sim 0.4 \mathrm{eV}$. Thus, the main photodissociation products were slow (low energy) $\mathrm{H}$ fragments in correlation with both singlet vinylidene $\left[\mathrm{CH}_{2} \mathrm{C}\left(\tilde{X}{ }^{1} A_{1}\right)\right]$ and singlet acetylene $\left[\mathrm{CHCH}\left(\tilde{X}^{1} \Sigma_{g}^{+}\right)\right]$. A minor contribution of triplet acetylene $\left[\mathrm{CHCH}\left(\tilde{A}^{3} B_{2}\right)\right]$ was also found. The finding of ground state vinylidene and acetylene as the main products is consistent with the PECs of Fig. 1 and 2, which, for relatively low energies in the first two excited states of VR, predict internal conversion to the ground state as the dominant dissociation mechanism. Most probably excitation at $\lambda=243 \mathrm{~nm}(5.10 \mathrm{eV})$ is not enough to reach the $\tilde{C}^{2} A^{\prime}-\tilde{B}^{2} A^{\prime \prime}$ CI indicated in Fig. 1a, which would have made it possible to detect excited triplet vinylidene, $\mathrm{CH}_{2} \mathrm{C}\left(\tilde{A}^{3} B_{2}\right)$. The experimental detection of a small amount of excited triplet acetylene might indicate that a CI between the $\tilde{B}^{2} A^{\prime \prime}$ and $\tilde{C}^{2} A^{\prime}$ states of VR similar to that of pathway (1) could exist also for pathway (2), but at lower energy.

In the photodissociation experiments by Bañares and coworkers at $\lambda=199 \mathrm{~nm},{ }^{27}$ of relatively hot radicals (i.e., with substantial internal energy), produced by in situ photolysis of vinyl iodide in the interaction zone, the $\mathrm{H}$-atom TED measured also exhibited a Boltzmann-type shape with a peak at $\sim 0.3 \mathrm{eV}$, being very similar to the distribution of the main contribution found in ref. 26. The likely electronic state excited in VR at $199 \mathrm{~nm}$ is $\tilde{C}^{2} A^{\prime}$, the third excited state, which is not reached at $243 \mathrm{~nm}$. From estimates of the maximum energy available for the fragments, ${ }^{27}$ the TED measured at $199 \mathrm{~nm}$ is consistent with $\mathrm{H}$ fragments in correlation with vinylidene both in the ground $\mathrm{CH}_{2} \mathrm{C}\left(\tilde{X}^{1} A_{1}\right)$ and first excited $\mathrm{CH}_{2} \mathrm{C}\left(\tilde{A}^{3} B_{2}\right)$ states, and also with acetylene in the ground state $\mathrm{CHCH}\left(\tilde{A}^{3} B_{2}\right)$. The PECs shown in Fig. 1a and 2a qualitatively explain this finding. Indeed, the excitation energy corresponding to $199 \mathrm{~nm}$ $(6.23 \mathrm{eV})$ plus the internal energy inherited by the VR in its generation seems to be enough to overcome the exit barrier of the $\tilde{C}^{2} A^{\prime}$ state in Fig. 1a, making possible the formation of $\mathrm{CH}_{2} \mathrm{C}\left(\tilde{A}^{3} B_{2}\right)$ fragments through a slow predissociation involving an efficient intramolecular vibrational energy redistribution (IVR) that would produce a Boltzmann-like distribution. Internal conversion to highly excited rovibrational levels of electronic states lower than $\tilde{C}^{2} A^{\prime}$, followed by a slow dissociation in the ground state, would produce $\mathrm{CH}_{2} \mathrm{C}\left(\tilde{X}^{1} A_{1}\right)$ fragments that again would give rise to a Boltzmann-type shape of the $\mathrm{H}$ atom TED. Fig. 2a, however, suggests that the excitation energy would not be above the $\tilde{C}^{2} A^{\prime}$ asymptote, and thus the formation of excited acetylene $\mathrm{CHCH}\left(\tilde{A}^{3} B_{2}\right)$ would not be possible. Only a slow internal conversion followed by a final dissociation on the ground state would give ground state acetylene.

The photodissociation experiments by Lee and co-workers at $\lambda=193 \mathrm{~nm}^{28}$ increase the excitation energy with respect to the $199 \mathrm{~nm}$ experiment. Since VR photodissociation in this case is a secondary process, after ethylene photodissociation, vinyl is produced with some initial internal energy before absorbing the photon of $193 \mathrm{~nm}$. Thus, it is estimated that energies of the
$\tilde{C}^{2} A^{\prime}$ state above the exit barrier are populated, and even the $\tilde{D}{ }^{2} A^{\prime}$ state (the 3 s Rydberg state) could be reached. From the PECs shown in Fig. 1a and 2a, this would be consistent with the formation of vinylidene and acetylene fragments in both the ground and first excited states. Indeed, as concluded in ref. 28, the position of the peak of the TED associated with the VR photodissociation appears to suggest that the production of excited triplet $\mathrm{CH}_{2} \mathrm{C}$ and $\mathrm{CHCH}$ could be an important dissociation pathway.

In Fig. 3 the PECs associated with pathway (3) are shown along with the nonadiabatic couplings between the different electronic states. To the best of our knowledge, no photodissociation experiments on this pathway have been reported. The PECs of Fig. 3a display an interesting and distinct feature as compared with those of Fig. 1a and 2a: the ground state of VR is much deeper for pathway (3), and its asymptote is thus located at remarkably higher energy. Indeed, the asymptote of the $\tilde{X}^{2} A^{\prime}$ state is at similar energy as the asymptote of the first excited $\tilde{A}^{2} A^{\prime \prime}$ state, above $7 \mathrm{eV}$. This implies that dissociation into $\mathrm{CH}_{2}+$ $\mathrm{CH}$ fragments implies rather high excitation energies, reaching at least nearly $8 \mathrm{eV}$, which is not surprising since this channel requires breaking a double bond. This involves populating the 3s $\left(\tilde{D}^{2} A^{\prime}\right)$ and $3 \mathrm{p}\left(\tilde{E}^{2} A^{\prime}\right)$ Rydberg states. Interestingly, the PECs of Fig. 3a predict that excitation of the three first excited states below $8 \mathrm{eV}$ will not produce dissociation through pathway (3), and only dissociation through the other two pathways will be possible.

\subsection{Two-dimensional representation of the vinyl radical}

A two-dimensional representation of VR has been used in order to explore the migration path of a $\mathrm{H}$ atom from the $\mathrm{C}_{\beta} \mathrm{H}_{2}$ group to the $\mathrm{C}_{\alpha} \mathrm{H}$ one. Fig. 4 displays the two coordinates $R$ and $\theta$ of this representation. The PECs corresponding to the ground and excited electronic states of VR along the $R$ coordinate are calculated for several angles in the range $\left[0,90^{\circ}\right]$, since the present 2D representation is symmetrical with respect to $\theta=90^{\circ}$. Specifically, the values of the angles for which calculations have been carried out are $\theta=10^{\circ}, 20^{\circ}, 30^{\circ}, 40^{\circ}, 50^{\circ}, 60^{\circ}$, $70^{\circ}, 75^{\circ}, 80^{\circ}, 85^{\circ}, 90^{\circ}$, and the equilibrium angle (i.e., the angle corresponding to the potential-energy minimum) $\theta=36.77^{\circ}$. It is noted that $a b$ initio $2 \mathrm{D}$ representations of the potentialenergy surfaces and nonadiabatic couplings have been previously reported for other radicals like $\mathrm{CH}_{3},{ }^{53}$ and $\mathrm{CH}_{3} \mathrm{O}$ and $\mathrm{CH}_{3} \mathrm{~S} .{ }^{54}$ In Fig. 5 the calculated PECs are displayed for different values of $\theta$.

The variation of the PECs with the angle $\theta$ shows several interesting features. The minimum energy configuration for

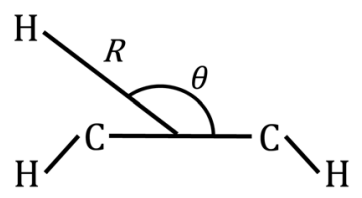

Fig. 4 Scheme of the Jacobi coordinates $R$ and $\theta$ used in the twodimensional representation of the vinyl radical, associated with the $\mathrm{H}$-atom migration. 

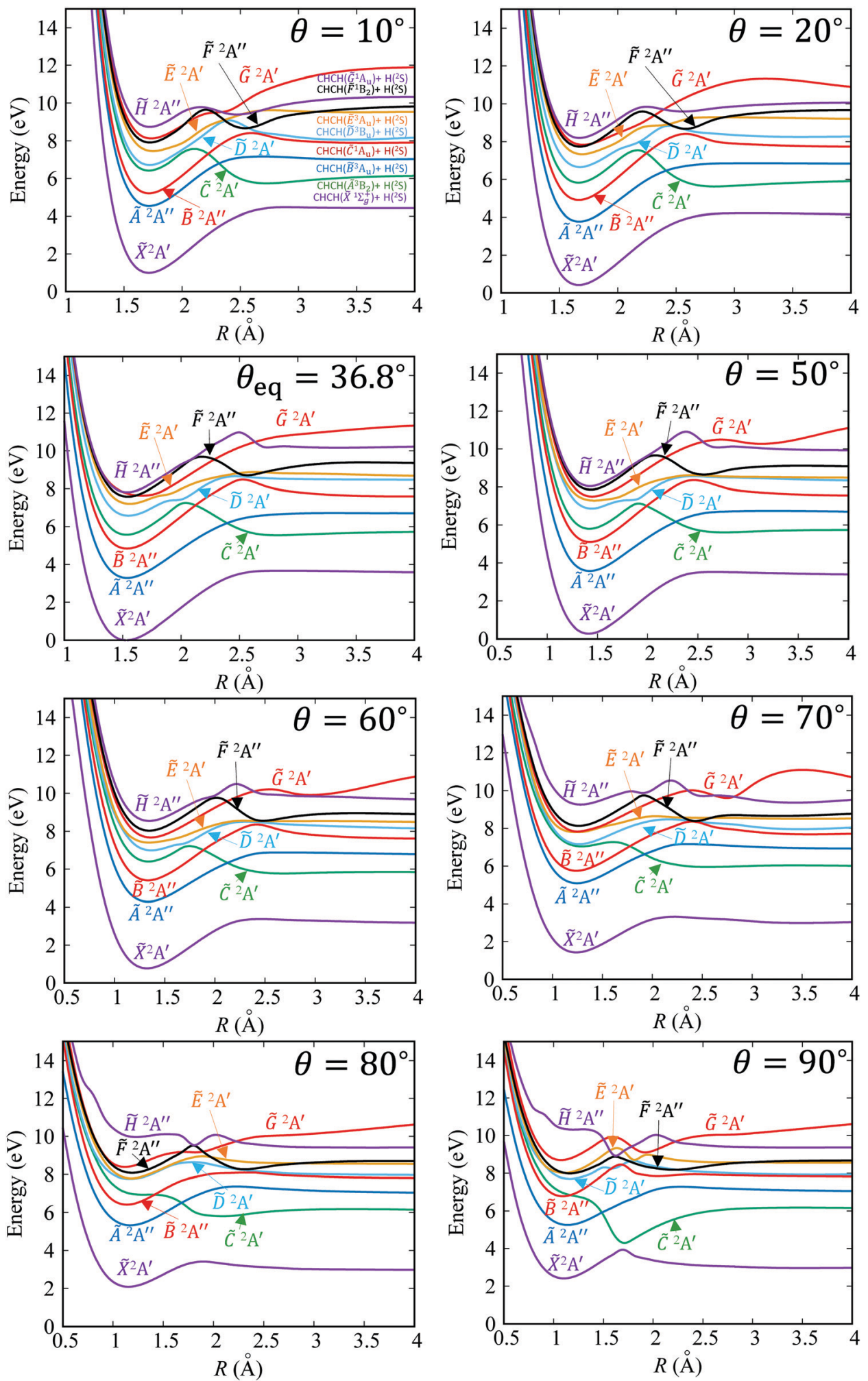

Fig. 5 Potential-energy curves of the different electronic states along the $R$ coordinate (see Fig. 4) calculated for different values of the angle $\theta$ in the range $\left[0,90^{\circ}\right]$.

the ground and most of the excited states is $\theta=36.77^{\circ}$ (and the equivalent configuration $\theta=143.23^{\circ}$ ). In general, the different electronic states keep the same relative positions of their energy minima as the angle changes. This holds for the first five electronic states, and even for the minimum of the $\tilde{H}$ state, which is typically the highest one. However, the relative 
position of the minima of the $\tilde{E}, \tilde{F}$, and $\tilde{G}$ states changes with $\theta$. In particular, the minimum of the $\tilde{F}$ state can be above the minima of the $\tilde{E}$ and $\tilde{G}$ states, like for $\theta=50^{\circ}$, or below those minima, as for $\theta=90^{\circ}$.

The most interesting finding of Fig. 5 is that several possible conical intersections are developed between the different electronic states in different angular regions. As a result, exit barriers to dissociation appear and disappear as $\theta$ varies. Examples of those CIs are between the $\tilde{C}$ and $\tilde{D}$ states near $R=2.0 \AA$ at angles in the region of $\theta=30^{\circ}-60^{\circ}$, between the states $\tilde{B}$ and $\tilde{F}$ near $R=2.5 \AA$, and between the states $\tilde{F}$ and $\tilde{H}$ around $R=1.8 \AA$ at angles in the region $\theta>60^{\circ}$. The $C_{2 \mathrm{v}}$ configuration associated with $\theta=90^{\circ}$ is an especially interesting case, since several additional CIs arise. We can see in the figure that a CI is developed between the $\tilde{D}$ and $\tilde{E}$ states near $R=1.8 \AA$, between the $\tilde{E}$ and $\tilde{G}$ states near $R=2.0 \AA$, and between the $\tilde{X}$ and $\tilde{C}$ states near $R=1.7 \AA$. This latter CI is very similar to the CI found between the $\tilde{X}$ state and the 3s Ryberg state in the ethyl radical for the corresponding bridged $C_{2 \mathrm{v}}$ configuration, which is very similar to the present $C_{2 \mathrm{v}}$ structure of VR. ${ }^{51}$ The presence of the above CIs and barriers determines how photodissociation will proceed when the different electronic states are excited. The existence of multiple CIs typically implies fast hydrogen dissociation in the states connected by the CIs. By contrast, exit barriers are associated with delays in the $\mathrm{H}$-atom elimination. Fig. 5 reflects a rich topography of the potentialenergy landscape, where the specific mechanism of hydrogen fragmentation will be very much dependent on the potential energy region accessed upon photoexcitation.

In previous works on the hydrogen fragmentation in the ethyl radical, fast $\mathrm{H}$ atoms were observed dissociating from the $\mathrm{C}_{\beta} \mathrm{H}_{3}$ group of the radical after excitation to the 3s Rydberg state. ${ }^{27,50,51} \mathrm{Ab}$ initio calculations were able to find the origin of this site-specific $\mathrm{H}$-atom elimination in the presence of a CI between the $3 \mathrm{~s}$ and the ground state of ethyl. ${ }^{51}$ This CI would arise at the bridged $C_{2 \mathrm{v}}$ configuration of ethyl, where one hydrogen atom of the $\mathrm{C}_{\beta} \mathrm{H}_{3}$ group would be located equidistant between the two remaining $\mathrm{CH}_{2}$ groups (in a configuration equivalent to that of $\theta=90^{\circ}$ in Fig. 4). Dissociation through this $\mathrm{CI}$ would generate the fast $\mathrm{H}$ fragments. The similarity of the ethyl $C_{2 \mathrm{v}}$ configuration to the equivalent one of VR, namely the planar bridged structure of $C_{2 \mathrm{v}}$ symmetry, and the presence of a similar CI between the gound and the $\tilde{C}$ state of VR at this bridged structure, leads us to think that a similar site-specific $\mathrm{H}$-atom elimination producing fast hydrogen fragments might also occur in the case of VR upon excitation of the $\tilde{C}$ state. This point will be discussed in more detail below.

It is interesting to analyze the behavior of the different electronic states along the angular coordinate $\theta$, since it provides information on the $\mathrm{H}$-atom migration from one carbon atom to the other one. The corresponding PECs are displayed in Fig. 6. The top of the barrier of the $\tilde{X}^{2} A^{\prime}$ electronic state corresponds to the planar saddle point associated with the bridged $C_{2 \mathrm{v}}$ structure of VR. The energy of this saddle point is found to be $\sim 2.41 \mathrm{eV}$ in the present calculations, in good agreement with the energy of $\sim 17800 \mathrm{~cm}^{-1}(\sim 2.21 \mathrm{eV})$ found

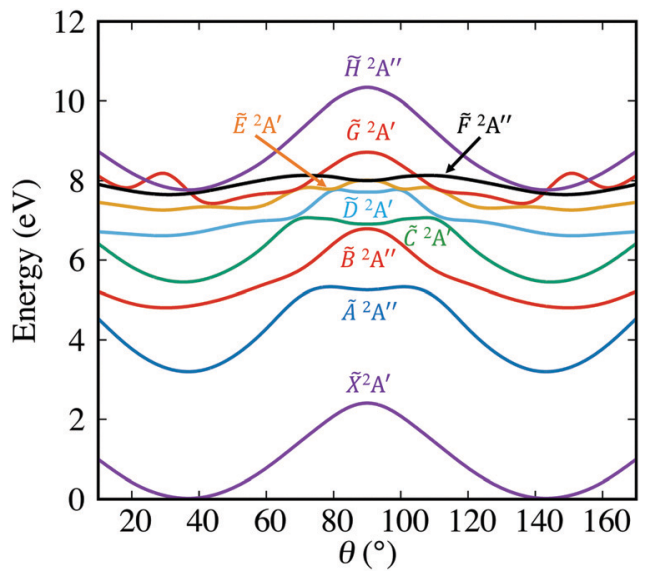

Fig. 6 Potential-energy curves of the different electronic states along the $\theta$ coordinate (see Fig. 4). The curves are obtained by selecting the energy values of each state corresponding to the $R$ distance for which the ground state $\tilde{X}^{2} A^{\prime}$ reaches the minimum energy at each angle $\theta$.

in previous simulations. ${ }^{21,39}$ The lowest-lying $C_{2 \mathrm{v}}$ saddle point lies much lower in energy, at $\sim 1700 \mathrm{~cm}^{-1}(\sim 0.21 \mathrm{eV}){ }^{39}$ The high-lying planar saddle point is involved in the in-plane hydrogen migration, and is expected to play a much more significant role in the 1,2 hydride shift tunneling dynamics than the non-planar saddle point (located at similar energy as the planar one), since the latter involves a larger atomic rearrangement.

Concerning the excited electronic states, Fig. 6 shows that almost all of them exhibit potential wells in the same angular region as the $\tilde{X}^{2} A^{\prime}$ state (the only exception is $\tilde{G}{ }^{2} A^{\prime}$ ). This implies a difference with the ethyl radical, where the two first excited states, the $3 \mathrm{~s}$ and $3 \mathrm{p}_{z}$ Ryberg states, showed a single potential well at the bridged $C_{2 \mathrm{v}}$ geometry $\left(\theta=90^{\circ}\right) .{ }^{47}$ Interestingly, several excited states display a relatively low barrier (remarkably lower than that of the ground state), in particular the states $\tilde{D}^{2} A^{\prime}, \tilde{E}^{2} A^{\prime}, \tilde{F}^{2} A^{\prime \prime}$, and $\tilde{G}^{2} A^{\prime}$. Such low barriers are expected to greatly favor in-plane hydrogen migration.

As noted above, the presence of a CI between the $\tilde{X}^{2} A^{\prime}$ and $\tilde{C}^{2} A^{\prime}$ states of VR at the $C_{2 \mathrm{v}}$ bridged configuration (see the $\theta=$ $90^{\circ}$ panel of Fig. 5) suggests the possibility of a site-specific $\mathrm{H}^{-}$ atom elimination producing fast hydrogen fragments (correlating with acetylene fragments), upon excitation of the $\tilde{C}^{2} A^{\prime}$ state, similarly as found in the ethyl radical when exciting the $3 \mathrm{~s}$ Rydberg state. However, in previous experiments on vinyl photodissociation at an excitation wavelength of $199 \mathrm{~nm},{ }^{27}$ where the $\tilde{C}^{2} A^{\prime}$ state was expected to be populated, no significant intensity of fast $\mathrm{H}$ fragments was found in the TED, which in principle discarded the occurrence of the site-specific elimination mechanism at this excitation wavelength. It is correct that such a mechanism is favored in ethyl by the fact that the potential-energy minimum of the 3s Rydberg state is located at the bridged structure where the CI with the ground state occurs, while Fig. 6 shows that the $\tilde{C}^{2} A^{\prime}$ state of vinyl exhibits a barrier at the bridged configuration where the CI with $\tilde{X}^{2} A^{\prime}$ arises. Nonetheless, the $\tilde{C}^{2} A^{\prime}$ barrier is not very high, 
and with enough excitation energy (probably reached with an excitation wavelength shorter than $199 \mathrm{~nm}$ ) it could be overcome, making possible the observation of the site-specific hydrogen elimination mechanism. It is noted, however, that in the hydrogen elimination after the secondary photodissociation of vinyl at $193 \mathrm{~nm},{ }^{28}$ fast $\mathrm{H}$ fragments were not observed either. This might be due to the fact that the competing mechanism involving the direct $\mathrm{H}$-atom elimination to produce $\mathrm{CH}_{2} \mathrm{C}$ fragments is more efficient in the case of VR, at least in these experimental conditions. Investigating further experimentally the possibility of site-specific $\mathrm{H}$-atom elimination in vinyl photodissociation should be very interesting.

\section{Conclusions}

Photodissociation of the vinyl radical is investigated by means of high-level MRCI $a b$ initio calculations. Three dissociation pathways $\mathrm{CH}_{2} \mathrm{CH} \rightarrow \mathrm{CH}_{2} \mathrm{C}+\mathrm{H}, \mathrm{CH}_{2} \mathrm{CH} \rightarrow \mathrm{CHCH}+\mathrm{H}$, and $\mathrm{CH}_{2} \mathrm{CH} \rightarrow \mathrm{CH}_{2}+\mathrm{CH}$ are studied by calculating the potentialenergy curves associated with the ground and several excited electronic states along the dissociating bond distance corresponding to each pathway, and the nonadiabatic couplings connecting the different states. The calculated potential curves are used to rationalize the main findings of the available experiments on the photodissociation of vinyl. The experiments are carried out at different excitation wavelengths, and thus reach different excited electronic states. The shape of the $a b$ initio potential-energy curves along with the nonadiabatic couplings found is able to explain, at least qualitatively, the observations of the different experiments.

In addition to the one-dimensional representation used for the above three fragmentation pathways, a two-dimensional representation is also adopted, consisting of two Jacobi coordinates, a distance and an angle, of one of the hydrogen atoms of the $\mathrm{C}_{\beta} \mathrm{H}_{2}$ group. Such a representation provides more detailed information on the $\mathrm{CH}_{2} \mathrm{CH} \rightarrow \mathrm{CHCH}+\mathrm{H}$ fragmentation pathway, as well as on the in-plane hydrogen migration from one carbon atom to the other one through a high-lying transition state. The potential-energy surfaces of the electronic states calculated in this two-dimensional representation exhibit a rich topography where several conical intersections, nonadiabatic couplings leading to predissociation, and exit barriers to dissociation, arise and disappear as the energy and the coordinate region changes. This picture thus reflects a very complex photodissociation dynamics, with very different fragmentation mechanisms depending on the energy and the specific electronic state excited. In this sense, analysis of the behavior of the different electronic states along the angular coordinate reveals that the mechanism of in-plane hydrogen migration might be greatly favored in some excited states, which display very low potential barriers associated with the transition state. The calculated potential-energy surfaces also suggest the possible occurrence of a site-specific elimination mechanism of a hydrogen atom from the $\mathrm{C}_{\beta} \mathrm{H}_{2}$ group, very similar to that previously found for the ethyl radical.

\section{Conflicts of interest}

There are no conflicts of interest to declare.

\section{Acknowledgements}

This research has been carried out within the Unidad Asociada Química Física Molecular between the Departamento de Química Física of Universidad Complutense de Madrid and CSIC. This work was funded by the Ministerio de Ciencia e Innovación (MICINN, Spain), Grant No. PGC2018-096444-B-I00 and Grant No. PID2019-107115GB-C21. A. B. acknowledges funding from the I-COOP program from CSIC, Grant No. COOPB20364, which made possible a research stay at Instituto de Física Fundamental, CSIC. This project has received funding from the European Union's Horizon 2020 Research and Innovation Programme under the Marie Skłodowska-Curie grant agreement No 872081. The Centro de Supercomputación de Galicia (CESGA, Spain) is acknowledged for the use of its resources.

\section{References}

1 P. R. Westmoreland, A. M. Dean, J. B. Howard and J. P. Longwell, J. Phys. Chem., 1989, 93, 8171.

2 J. A. Miller, R. J. Kee and C. K. Westbrook, Annu. Rev. Phys. Chem., 1990, 41, 345.

3 B. K. Carpenter, J. Phys. Chem., 1995, 99, 9801.

4 R. K. Janev and D. Reiter, Phys. Plasmas, 2004, 11, 780.

5 R. A. West, D. F. Strobel and M. G. Tomasko, Icarus, 1986, 65, 161-217.

6 W. H. Ip, Astrophys. J., 1990, 362, 354.

7 A. Fahr, P. S. Monks, L. J. Stief and A. H. Laufer, Icarus, 1995, 116, 415.

8 G. R. Gladstone, M. Allen and Y. L. Yung, Icarus, 1996, 119, 1.

9 P. N. Romani, Icarus, 1996, 122, 233-241.

10 W. C. Gardiner, Jr, Combustion Chemistry, Springer, New York, 1984.

11 A. Fahr and A. H. Laufer, J. Phys. Chem., 1988, 92, 7229-7232.

12 D. L. Baulch, C. J. Cobos, R. A. Cox, C. Esser, P. Frank, T. Just, J. A. Kerr, M. J. Pilling, J. Troe, R. W. Walker and J. Warnatz, J. Phys. Chem. Ref. Data, 1992, 21, 411-734.

13 H. E. Hunziker, H. Kneppe, A. D. McLean, P. Siegbahn and H. R. Wendt, Can. J. Chem., 1983, 61, 993-995.

14 A. Fahr, A. Laufer, R. Klein and W. Braun, J. Phys. Chem., 1991, 95, 3218-3224.

15 A. Fahr, P. Hassanzadeh and D. B. Atkinson, Chem. Phys., 1998, 236, 43.

16 C. D. Pibel, A. McIlroy, C. A. Taatjes, S. Alfred, K. Patrick and J. B. Halpern, J. Chem. Phys., 1999, 110, 1841-1843.

17 L. Letendre, D. K. Liu, C. D. Pibel, J. B. Halpern and H. L. Dai, J. Chem. Phys., 2000, 112, 9209-9212.

18 M. B. Pushkarsky, A. M. Mann, J. S. Yeston and C. B. Moore, J. Chem. Phys., 2001, 115, 10738. 
19 M. Shahu, C.-H. Yang, C. D. Pibel, A. McIlroy, C. A. Taatjes and J. B. Halpern, J. Chem. Phys., 2002, 116, 8343.

20 A. Carvalho, G. Hancock and M. Saunders, Phys. Chem. Chem. Phys., 2006, 8, 4337-4346.

21 K. Tanaka, M. Toshimitsu, K. Harada and T. Tanaka, J. Chem. Phys., 2004, 120, 3604-3618.

22 L. Letendre, D.-K. Liu, C. D. Pibel, J. B. Halpern and H.-L. Dai, J. Chem. Phys., 2000, 112, 9209.

23 Y.-J. Wu, M.-Y. Lin, B.-M. Cheng, H.-F. Chen and Y.-P. Lee, J. Chem. Phys., 2008, 128, 204509.

24 H. Kanamori, Y. Endo and E. Hirota, J. Chem. Phys., 1990, 92, 197-205.

25 F. Dong, M. Roberts and D. J. Nesbitt, J. Chem. Phys., 2007, 127, 1.

26 M. Ahmed, D. S. Peterka and A. G. Suits, J. Chem. Phys., 1999, 110, 4248-4253.

27 D. V. Chicharro, A. Zanchet, A. Bouallagui, L. Rubio-Lago, A. García-Vela, L. Bañares and S. Marggi Poullain, Phys. Chem. Chem. Phys., 2021, 23, 2458-2468.

28 B. A. Balko, J. Zhang and Y. T. Lee, J. Chem. Phys., 1992, 97, 935-942.

29 L. B. Harding, J. Am. Chem. Soc., 1981, 103, 7469.

30 L. B. Harding, A. F. Wagner, J. M. Bowman, G. C. Schatz and K. Christoffel, J. Chem. Phys., 1982, 86, 4312.

31 M. Dupuis and J. J. Wendoloski, J. Chem. Phys., 1984, 80, 5696. 32 M. N. Paddon-Row and J. A. Pople, J. Phys. Chem., 1985, 89, 2768. 33 L. A. Curtiss and J. A. Pople, J. Chem. Phys., 1988, 88, 7405. 34 J.-H. Wang, H.-C. Chang and Y.-T. Chen, Chem. Phys., 1996, 206, 43.

35 A. M. Mebel, Y.-T. Chen and S.-H. Lin, Chem. Phys. Lett., 1997, 275, 19.

36 A. R. Al Derzi, S. Fau and R. J. Bartlett, J. Phys. Chem. A, 2003, 107, 6656.

37 G. V. Milnikov, T. Ishida and H. Nakamura, J. Phys. Chem. A, 2006, 110, 5430.

38 D. J. Nesbitt and F. Dong, Phys. Chem. Chem. Phys., 2008, 10, 2113.

39 A. R. Sharma, J. M. Bowman and D. J. Nesbitt, J. Chem. Phys., 2012, 136, 034305.
40 A. R. Sharma, B. J. Braams, S. Carter, B. C. Shepler and J. M. Bowman, J. Chem. Phys., 2009, 130, 174301.

41 L. Chen, K. Shao, J. Chen, M. Yang and D. H. Zhang, J. Chem. Phys., 2016, 144, 194309.

42 H.-G. Yu, H. Song and M. Yang, J. Chem. Phys., 2017, 146, 224307.

43 J. Šmydke, C. Fábri, J. Sarka and A. G. Császár, Phys. Chem. Chem. Phys., 2019, 21, 3453-3472.

44 X.-G. Wang and T. Carrington, Jr., J. Chem. Phys., 2020, 152, 204311.

45 A. Matsugi, J. Phys. Chem. Lett., 2013, 4, 4237-4240.

46 P. J. Knowles and H.-J. Werner, Theor. Chim. Acta, 1992, 84, 95.

47 H.-J. Werner, Mol. Phys., 1996, 89, 645.

48 H.-J. Werner and P. J. Knowles, J. Chem. Phys., 1985, 82, 5053.

49 H.-J. Werner, P. J. Knowles, R. Lindh, M. Schütz, P. Celani, T. Korona, F. R. Manby, G. Rauhut, R. D. Amos, A. Bernhardsson, A. Berning, D. L. Cooper, M. J. O. Deegan, A. J. Dobbyn, F. Eckert, C. Hampel, G. Hetzer, A. W. Lloyd, S. J. McNicholas, W. Meyer, M. E. Mura, A. Nicklass, P. Palmieri, R. Pitzer, U. Schumann, H. Stoll, A. J. Stone, R. Tarroni, T. Thorsteinsson and M. Wang, MOLPRO, version 2012, a package of ab initio programs, see: http:/www.molpro.net.

50 D. V. Chicharro, S. Marggi Poullain, A. Zanchet, A. Bouallagui, A. García-Vela, M. L. Senent, L. Rubio-Lago and L. Bañares, Chem. Sci., 2019, 10, 6494-6502.

51 S. Marggi Poullain, D. V. Chicharro, A. Zanchet, L. RubioLago, A. García-Vela and L. Bañares, Phys. Chem. Chem. Phys., 2019, 21, 23017-23025.

52 S. Marggi Poullain, L. Rubio-Lago, D. V. Chicharro, A. Bouallagui, A. Zanchet, O. Yazidi, A. García-Vela and L. Bañares, Mol. Phys., 2022, 120:1-2, e1984598.

53 A. Zanchet, L. Bañares, M. L. Senent and A. García-Vela, Phys. Chem. Chem. Phys., 2016, 18, 33195-33203.

54 A. Bouallagui, A. Zanchet, O. Yazidi, N. Jadane, L. Bañares, M. L. Senent and A. García-Vela, Phys. Chem. Chem. Phys., 2017, 19, 31245-31254. 